\title{
The Effect of Network Communication on Children's Social Creativity
}

\author{
Enhe Hao, Xiaorong Zhang, Zhi Jing, Chuanhua Gu* and Jingjing Song \\ Key Laboratory of Adolescent Cyberpsychology and Behavior(Central China Normal University), Ministry of \\ Education, Wuhan 430079, China \\ *Corresponding Author, guchuanhua2008@yahoo.cn
}

\begin{abstract}
Social creativity is a special sort of creativity in the domain of social interaction or activities, and it can be classified into two types: stable trait creativity and instable state creativity. To investigate the effect of network communication on social creativity including trait creativity and state creativity, the Open Questionnaire of Social Creativity for Primary School Children, the Adolescents' Internet Use Preference Questionnaire, and the Open Questionnaire of Social Creativity for Primary School Children were administered to 650 primary school children randomly selected from grade 3 to grade 6 . The result showed that significant positive correlation existed between network communication and trait social creativity instead of state social creativity; furthermore, network communication could predict trait creativity instead of state creativity significantly. This indicates that trait creativity and state creativity may be relatively independent, and network communication affects individual's consistent creative tendencies.
\end{abstract}

Index Terms - social creativity, trait creativity, state creativity, network communication, primary school children

\section{Introduction}

It is commonly believed that creativity is the ability to produce innovative, unique and useful perspective or product [1]. At the 1950 meeting of the American Psychological Association, Guilford argued that creativity was an understudied yet promising field in his presidential address. Since then, creativity research has made great progress in the past half century.

\section{A. Network Communication and Creativity}

In recent years, the systematic perspective of creativity has been accepted by more and more researchers. In this view, creation is thought as an activity jointly influenced by a variety of factors including creators' knowledge, cognitive characteristics, personality tendencies, and environment [2]. Creativity investment theory illustrates the system perspective of creativity typically. It emphasizes the effect of environment, considering that the relationship between the individual and the environment is similar to that between the investors and the stock market; and then it proposed six types of interactive resources that are invested in creation by the creator and will affect creative performance: intellectual abilities, knowledge, thinking styles, personality, motivation, and environment [1].

In recent years Internet has become an indispensable environment for children. It has a profound impact on personal life. According to the report from China Internet Network Information Center (CNNIC), the number of Internet users in China has reached 538 million by the end of June 2012, and Internet penetration rate is $39.9 \%$ [3]. As one way of Internet use, network communication has profoundly affected the social development of Internet users. However, the results are inconsistent. Some researchers applied "rich get richer" model to explain Internet social networking of users, considering it provided more day-to-day interaction channels so that it would enhance the individual's social resources [4]. Other researchers who believed social interaction on the Internet would replace day-to-day social interaction with family and friends preferred social displacement hypothesis, believing it had a negative impact on mental health of Internet users [5].

How does the Internet use, particularly network communication, affect a person's creativity? Large numbers of studies have investigated the effect of general environments including family, school, and even organized environment on creativity. With the growing popularity of Internet, it is likely to affect the development of children's creativity. Although researchers have explored this area [6], it is still unclear how the Internet affects the development of creativity in different areas and in different forms. This implies that further studies in this field are needed.

\section{B. Social Creativity}

Since the 1980s, many researchers have proposed that creativity is domain-specific, that is, creativity in different areas is different. Among these theories, Gardner's theory of Multiple Intelligences and Csikszentmihalyi's system perspective of creativity is most typical. Social creativity is a special sort of creativity in social interaction or social activities where a person proposes or solves social problems in the novel, special, appropriate and valid approaches, and it is correspondent to artistic creativity or scientific creativity [7]. In fact, in the Theory of Multiple Intelligences, interpersonal intelligence is regarded as the outstanding performance of social creativity. Mouchiroud et al. (2002) carried out a study to investigate social creativity of primary school children in France, pointing out that social creativity was a relatively independent structure. Gu et al. $(2007,2008,2010)$ also found that children's social creativity was a more coherent and integrated structure in the context of Chinese culture through a

\footnotetext{
* This work is partially supported by National Youth Funding Grant "Cross-cultural Study of Children's Social Creativity: Based on the Comparison between Eastern Culture and Western Culture" (CDA110106) to G. Chuanhua.
} 
series of studies about social creativity of primary school children [8-10].

Compared to artistic creativity and scientific creativity, social creativity may have a closer association with network communication. Prior studies have found that social creativity is closely associated with a person's social activities and interactive experience $[11,12]$. Undoubtedly it is significant to explore the effect of network communication on children's social creativity.

\section{Trait Creativity and State Creativity}

Hennessey and Amabile (2010) reviewed the creativity research of the past half century, and divided them into seven levels or types: neurological, affect/cognitive/training, individual/personality, groups, social environment, cultural/social and systems approach [13]. Just as mentioned above, the systematic perspective of creativity considers creativity is influenced not only by individual personality but also by environment or situation. Creative performance of an individual may be stable orientation, or may be the transient state affected by the environment. In the present study, social creativity is divided into trait creativity and state creativity, and their relationship with network communication is explored respectively, and furthermore, the influence of network communication on the two forms of social creativity is identified. As a status, state creativity can be regarded as a form of creativity. It refers to individual's performance in specific problem situations (often in a comparatively short period of time), which is influenced by temporary factors such as individual emotional state. On the contrary, trait creativity can be another form of creativity. It refers to individuals' consistent creative tendencies or qualities with relative stability or continuity.

According to the definition of trait creativity and state creativity, this study assumes that network communication, as the result of the interaction of individual factors and environmental factors, has significant positive correlation with both trait creativity and state creativity, however, it affects the two forms of social creativity to different extent because they are different in nature.

\section{Method}

\section{A. Participants}

Two representative primary schools were selected from Zhijiang City of Hubei Province through cluster sampling method, and 650 students from grade 3 to grade 6 participated in this study. Among these participants, boys were 306 and girls were $324 ; 188$ students from grade 3, 182 students from grade 4, 134 students from grade 5, and 146 students from grade 6 . The average age was 9.81 years old $(\mathrm{SD}=1.31$ years old).

\section{B. Measures}

1) The Adolescents' Internet Use Preference Questionnaire: This questionnaire was compiled by Cheng (2008), including 16 items which were separated into 3 dimensions: preference for information communication, preference for entertainment, and preference for information obtaining, and it can be used for children and adolescents from 9 to 14 years old [14]. The present research adopted the dimension of preference for information communication only to investigate children's online social interaction. The average score of the questionnaire ranged from 0 to 4 , and the higher score showed the higher preference. The internal consistency coefficient of the subscale or dimension in this research was 0.81 .

2) Social Creative Potential Questionnaire for Primary School Children: This questionnaire was compiled by Gu et al. (2008) to investigate children's trait social creativity, including 24 items which were divided into 6 dimensions: prestige, problem-solving ability, eminence, fortitude and emulousness, social intelligence, initiative and conscientiousness. The accumulated explanation rate of the 6 dimensions in exploratory factor analysis was 52.15\%. The Cronbach' $\alpha$ coefficient of the whole questionnaire was 0.87 , the split-half reliability coefficient was 0.81 , and the test-retest reliability coefficient was 0.63 . Hence the reliability of the questionnaire accorded with the psychometric demands. Meanwhile, the construction of the questionnaire was reasonable, having good construct validity. There were 3 choices for each item: completely unsuitable, partially suitable, and completely suitable. The average score of the questionnaire ranged from 0 to 4 , and the higher score represented the higher trait creativity [15]. The Cronbach' $\alpha$ coefficient of the questionnaire in the present research was 0.90 .

3) The Open Questionnaire of Social Creativity for Primary School Childre: This questionnaire was compiled by $\mathrm{Gu}$ and Zhou (2008) to investigate children's state social creativity. It includes 12 open interpersonal problems. Among them 9 problems were about hypothetical interpersonal interaction and 3 problems were about real interpersonal interaction. Children were asked to answer how to react to each problem, and required to give as many unique solutions to each problem as possible. The 12 problems included 3 typical social interaction situations: peer interaction, teacherstudent interaction and parent-child interaction. 8 postgraduates majoring in psychology scored each child's responses to each interpersonal problem on originality, fluency, flexibility, curiosity, complexity, risk-taking, appropriateness, and validity independently according to the same scoring criteria [9]. The Cronbach's $\alpha$ coefficient of the ratings was 0.93 .

\section{Procedure}

The questionnaires were administered to participants by trained postgraduates of psychology in each classroom according to the same instruction. The direction highlighted that the participants should answer the items truthfully and their answers would be kept secret. SPSS 17.0 were used to input and analyze the data. 


\section{Results}

A. Gender and grade difference in network communication, trait creativity, and state creativity

As showed in TABLE 1, boys were more likely to communicate with friends online than girls. State creativity of girls was higher than that of boys. Grade differences were found in network communication and trait creativity. Basically, children tended to communicate with others as they grew up gradually. The trend of trait creativity differed from that of state creativity. There was an obvious slump at grade four and grade five in trait creativity, whereas there was a gradual increase in state creativity.

TABLE 1 Gender and grade difference in children's network communication, trait creativity, and state creativity

\begin{tabular}{cccc}
\hline & Network & Trait creativity & State creativity \\
\hline communication & & \\
Female & $2.65 \pm 0.53$ & $2.95 \pm 0.37$ & $1.77 \pm 0.14$ \\
$\mathrm{~T}$ & $2.51 \pm 0.48$ & $2.99 \pm 0.44$ & $1.80 \pm 0.15$ \\
Grade 3 & $3.29^{* *}$ & -1.06 & $-3.02^{* *}$ \\
Grade 4 & $2.40 \pm 0.55$ & $3.05 \pm 0.43$ & $1.77 \pm 0.15$ \\
Grade 5 & $2.60 \pm 0.53$ & $2.90 \pm 0.39$ & $1.78 \pm 0.13$ \\
Grade 6 & $2.51 \pm 0.43$ & $2.90 \pm 0.45$ & $1.79 \pm 0.19$ \\
$\mathrm{~F}$ & $2.82 \pm 0.37$ & $3.01 \pm 0.35$ & $1.81 \pm 0.13$ \\
\hline Footnote: ${ }^{* *} \mathrm{p}<0.01,{ }^{* * *} \mathrm{p}<0.001$ & $6.63^{* * *}$ & 2.25 \\
\hline
\end{tabular}

B. Correlations between network communication, trait creativity, and state creativity

Positive correlation was found between network communication and trait creativity. However, there was no significant correlation between network communication and state creativity (see TABLE 2).

TABLE 2 Correlations between children's network communication, trait creativity, and state creativity

\begin{tabular}{clll}
\hline & 1 & 2 & 3 \\
\hline 1. Network communication & 1.00 & & \\
2. Trait creativity & $0.25^{* * *}$ & 1.00 & \\
3. State creativity & 0.04 & $0.10^{*}$ & 1.00 \\
\hline
\end{tabular}

Footnote: ${ }^{*} \mathrm{p}<0.05,{ }^{* * *} \mathrm{p}<0.001$

\section{The effect of network communication on social creativity}

The result from regression analysis of children's social creativity on network communication is shown in TABLE 3. It indicated that network communication could predict children's trait social creativity instead of state social creativity dramatically. It implied that network communication could affect trait social creativity rather than state social creativity.

TABLE 3 Regression analysis of children's social creativity on network communication

\begin{tabular}{ccccc}
\hline $\begin{array}{c}\text { Predict } \\
\text { variable }\end{array}$ & $\begin{array}{c}\text { Dependent } \\
\text { variable }\end{array}$ & F (1, 642) & B & t \\
\hline Network & Trait creativity & $43.00^{* * *}$ & $0.25^{* * *}$ & $6.56^{* * *}$ \\
communication & State creativity & 1.17 & 0.04 & 1.08 \\
\hline
\end{tabular}

Footnote: ${ }^{* * *} \mathrm{p}<0.001$

\section{Discussion}

This study found that network communication could significantly predict trait creativity, and it had significant impact on individual's social creativity tendencies. This confirms the assumption of the present study. This study also found that the prediction of network communication on state creativity was not significant, showing that network communication could not significantly affect the state creativity. This does not confirm the hypothesis.

Considering the impact of network communication on trait creativity, the results of this study are consistent with the previous findings in recent years. Related research showed that network communication could promote the development and performance of creativity. For example, Kock (2007) argued that the information exchange on the Internet would make information more ambiguous, which might be harmful to the fluency of information exchange. However, because of the ambiguity and lack of fluency, this information exchange could improve the individual creativity. An Individual can make use of his or her cognitive structure and spend more time to explain the information received. Clearly the process of using existing cognitive structure to assimilate network information benefits the development of individual creativity [6]. In other words, long-time network communication can affect an individual's creative personality tendencies.

However, as a sort of creative state in a particular situation, state creativity is directly affected by the problem situation, and has no close association with long-term network communication. In other words, network communication is more likely to affect children's creative personality or consistent creative tendencies rather than the state of creativity in a unique context. This creative state is more likely to be inspired by distinctive contexts. Some situations may promote creative expression, while other situations may inhibit creative expression. Shalley (1995) confirmed that inhibition of new products or ideas was more likely to occur in face-to-face communication. Because individuals may spend too much time and effort on day-to-day details in face-to-face situations, and too many non-verbal clues which regularly interacted with the information content would hinder the individual creativity, the face-to-face situation will eventually suppress individual creativity [16].

The results of this study also showed that there was lower but significant positive correlation coefficient between trait social creativity and state social creativity, indicating trait creativity and state creativity might be two relatively independent structures. In other words, they have both similarities and significant differences. Trait creativity is an unwavering creative tendency which is more likely formed by a relatively stable environment or a long-term lifestyle. People who possess higher trait creativity are more likely to face and solve a variety of problems in a new and unique way, but in specific contexts they may or may not exhibit higher creativity. Level of state creativity is straightforwardly associated with the characteristics of the situation. In specific contexts, people who have specific cognitive abilities and personality 
tendencies may be likely to exhibit a higher level of state creativity, and may also exhibit lower state creativity. To divide creativity (social creativity) into trait (social) creativity and state (social) creativity helps to explain the mechanism of individuals' creative achievements and to understand why the scales or tests focusing on the examination of state creativity are difficult to predict individuals' creative achievements.

\section{References}

[1] R.J. Sternberg and T.I. Lubart: "Investing in creativity". American Psychologist, vol. 51, no. 7, pp. 677-688, 1996.

[2] C. Gu: "The systematic perspective of creativity and its implications for education”. Educational Research and Experiment, vol. 3, pp. 51-55, 2005.

[3] Information on http://www.cnnic.cn/research/

[4] R. Kraut, M. Patterson, V. Lundmark, S. Kiesler, T. Mukhopadhyay, and W. Scherlis: "Internet Paradox". American Psychologist, vol. 53, no. 9, pp. 1017-1031, 1998.

[5] P.M. Valkenburg, A.P. Schouten and J. Peter: “Adolescents' identity experiments on the Internet". New Media \& Society, vol. 7, no. 3, pp. 383-402, 2005.

[6] N. Kock: "Media naturalness and compensatory encoding: the burden of electronic media obstacles is on senders". Decision Support Systems, vol. 44, pp. 175-187, 2007.

[7] C. Mouchiroud and T.I. Lubart: "Social creativity: a cross-secitonal study of 6-to 11-year-old children". International Journal of Behavioral Development, vol. 26, no. 1, pp. 60-69, 2002.
[8] C. Gu: "An experiment on the cultivation of social creativity tendency among elementary school children". Educational Research and Experiment, vol. 5, pp. 65-68, 2007.

[9] C. Gu and Z. Zhou: "On the relationship between social creative tendency and parenting style among primary school children". Psychological Development and Education, vol. 2, pp. 34-38, 2008.

[10] C. Gu, Y. Liu and Z. Zhou: "The internal consistency and domainspecificity of social creativity among primary school children". Psychological Science, vol. 33, no. 3, pp. 616-620, 2010.

[11] C. Gu, C. Huang and Z. Zhou: "The relationship between social creativity and social skill of primary school children and its implications for education". Educational Research and Experiment, vol. 2, pp. 56-59, 2008.

[12] C. Gu, Z. Zhou and M. Chong: "The relationship among social creativity, peer relationship and academic achievement of primary school children". Psychological Development and Education, vol. 3, pp. 20-25, 2009.

[13] B.A. Hennessey and T. M. Amabile: "Creativity". Annual Review of psychology, vol. 61, pp. 569-598, 2010.

[14] J. Cheng: "The Effect of Internet Use Preference, Information Skills on Adolescents' Academic Achievement". Doctoral Dissertation, Central China Normal University, 2008.

[15] C. Gu, Z. Zhou and J. Hu: "Psychometric analysis of social creative potential questionnaire for primary school children". Chinese Journal of Clinical Psychology, vol. 16, no. 4, pp. 340-343, 2008.

[16] C. E. Shalley: "Effects of coaction, expected evaluation, and goal setting on creativity and productivity". The Academy of Management Journal. vol. 38, pp. 483-503, 1995. 